\section{Research Article}

(C) 2021 Algethami et al.. This is an open access article licensed under the Creative Commons Attribution-NonCommercial 4.o International License (https://creativecommons.org/licenses/by-nc/4.o/)

Received: 8 March 2021 / Accepted: 23 June 2021 / Published: 8 July 2021

\title{
The Effect of Social Media Exposure on the Mental Health of Individuals with Disabilities during the Coronavirus 2019 Pandemic
}

\author{
Rasheed Khuwayshan Algethami ${ }^{1}$ \\ Turki Mahdi Alqarni \\ Fahmi Mustafa Albakour ${ }^{1}$ \\ 'Special Education Department, Taif University, \\ Al-Hawiyah, Taif, 21944, Saudi Arabia \\ ${ }^{2}$ Special Education Department, Najran University, \\ King Abdulaziz Rd, Najran, Saudi Arabia
}

DOI: https://doi.org/10.36941/jesr-2021-oo86

\begin{abstract}
The purpose of this study is to examine the mental health problems and social media exposure for individuals with disabilities, as well as explore whether this exposure could impact their mental health during the COVID19 epidemic period. A hundred and twenty individuals with disabilities participated in this cross-sectional study, conducted in Taif, Saudi Arabia. We used an online questionnaire built through the translation of two scales, including Generalized Anxiety Disorder-7 " GAD-7" and CES-D-10 scale assessing depression. The findings of this study underlined important messages for individuals with disabilities. First finding revealed that individuals with disabilities were almost half a day anxious, depressed, and frequently exposed to social media. Also, the information spread on social media had a significant effect on their anxiety level, but no significant effect was found on their depression level. Practical recommendations were highlighted to benefit these individuals during the COVID-19 pandemic period. We should use clear and accessible languages and health messages with individuals with disabilities. Health care providers should be exposed to an awareness program to reduce the racisms towards those individuals.
\end{abstract}

Keywords: Mental health problems, Social media, Persons with disabilities, COVID-19

\section{Introduction}

Since the description of the World Health Organization (WHO) about the outbreak of a new coronavirus (COVID-19) as "a Public Health Emergency of International Concern," many countries warned their population about this pandemic along with the increase of their safety and security by applying some restrictions (World Health Organization. 2020). For example, Saudi Arabia has applied effective restrictions, including pausing arrived and departing flights, forcing citizens to wear masks, and other restrictions to prevent the spread of this pandemic (Boretti, 2020).

This pandemic has not just spread among the people; it has also triggered the sharing of many news on social media platforms and numerous information among many communities. However, the 
World Health Organization's announcement of the outbreak has provided some messages to prevent misleading information about COVID-19 (World Health Organization. 2020). These messages were provided to different people, including the general population, health care workers, managers in health facilities, children in care, older adults, and people in the curfew (World Health Organization. 2020). Meaningful messages were also provided to people in general and people with disabilities related to the negative information on social media and its association with mental health problems. For instance, families and their children need to avoid watching or listening to fake news spreading in the public or social media, making children with disabilities more anxious, depressed, and angry during the curfew (Manganello, 2020). Therefore, while they are in the curfew period, they may be exposed to some types of social networks, following the news of COVID-19 outbreaks, which can potentially impact their mental health (Manganello, 2020).

\section{Literature Review}

\subsection{Implications of COVID-19 on Disabled Individuals}

The risks of the COVID-19 epidemic have inevitably reached people's psychological sides and have many negative consequences on their mental health (Alkhamees, Alrashed, Alzunaydi, Almohimeed, \& Aljohani, 2020). In other words, individuals who experience negative whose moods changed and who spend their time eating and sleeping are more likely to experience some types of mental illness such as anxiety, depression, and stress (Courtenay \& Perera, 2020).

Since the outbreak of COVID-19, individuals with intellectual, sensory, and physical disabilities have been experiencing disadvantageous implications, which have increased significantly with the threat of the COVID-19 pandemic (Armitage \& Nellums, 2020). 80\% of these individuals live in lowincome or middle-income countries, and, thus, they experience a lack of inclusive care during the COVID-19 pandemic (Tremmel, Myers, Brunow, \& Hott, 2020). Therefore, they need essential inclusive strategies to support them during the COVID-19 pandemic (Armitage \& Nellums, 2020).

Many schools have been suspended in 189 countries due to the COVID-19 pandemic since April 2020, which urged them to shift to distancing learning and online classes to teach students with and without disabilities (Patel, 2020). Although many educators believe that these online classes are the best alternatives for learning today, others have argued this type of education may negatively affect the mental health of students with disabilities (Tremmel et al., 2020).

Globally, the spread of COVID-19 has negatively impacted the lives of many students with disabilities in many countries (Tandon, 2020). For example, students with mental disabilities, whose number is almost eight million in India (UNICEF, 2020), have had significant challenges related to the lack of assistive technologies and inclusive education. Thus, staying at home has led them to feel frustrated and helpless (Tandon, 2020). However, students with disabilities in the United States of America receiving special education classes at schools reached seven million students (National Center for Education Statistics, 2020). The implications of the COVID-19 pandemic have shifted many special education classes to online classes, which deprived many students with learning disabilities, Down syndrome of necessary assistive technologies, and the special education assistances as parents cannot replace special education teachers (Tandon, 2020). Excessively, parents of individuals with disabilities have experienced elevated levels of stress and anxiety during this pandemic (Bartlett, Griffin, \& Thomson, 2020).

Additionally, most children with physical disabilities and pre-existing mental health face challenges in online learning, and this learning are just appropriate for students with visual or hearing impairments (Hill, 2020). Difficulties of distancing learning are associated with recreational activities, which are very frustrating for many students with physical disabilities (Armitage \& Nellums, 2020). Furthermore, students with autism spectrum disorders have experienced more issues in terms of social interactions and skills. For instance, applying social distancing, lack of access to outdoor activities, and lack of routines make students with ASD feel very anxious, stressed (Hill, 2020). 
The declaration of the COVID-19 outbreak has primarily been transmitted on many social platforms and TV news (Thelwall \& Levitt, 2020). This issue was noticed on Twitter; indeed, 55 tweets related to individuals with disabilities were identified in 285,471 re-tweets, and most of these re-tweets mentioned the term support for disabilities at the COVID-19 outbreak (Thelwall \& Levitt, 2020). Therefore, social networks can be beneficial tools for supporting meaningful activities for students with disabilities during pandemics (Caton \& Chapman, 2016; McNeil, Brna, \& Gordon, 2012).

The advantages of social media for individuals with disabilities were highlighted in many previous studies (Miller, 2017; Sweet, LeBlanc, Stough, \& Sweany, 2020). The social web can increase the interpersonal interactions of persons with disabilities, which help them shape identities and communities with likeminded people (Miller, 2017; Sweet et al., 2020) to obtain aids, confront bullying (Sweet et al., 2020) and represent their own stories and self-description (Gale \& Bolzan, 2016). The critical function of these stories can be used to overcome stereotypes and hidden segregations (Cocq \& Ljuslinder, 2020). Although social media appears to be a valuable space for persons with disabilities, it can be harmful to them through cyberbullying and hate-speak (Kowalski, Toth, \& Morgan, 2018).

Apart from hate speech on social media, the mental health of people with disabilities can be negatively affected (Dalton, Rapa, \& Stein, 2020). The outbreak entirely rules media and the social web, and people are exposed to a great deal of information with high levels of stress and anxiety (Dalton et al., 2020). They become overwhelmed by the amount of information transmitted by social networks on which they focus all their attention (Dalton et al., 2020). Additionally, they dread taking the treatment of pre-existing medical conditions for the elderly in hospitals during the COVID-19 pandemic. Moreover, many people with disabilities frequently experienced the threat of COVID-19 by exposing them to much misinformation through digital and social media (Goggin \& Ellis, 2020).

\section{The Present Study}

Given the importance of protecting the mental health of individuals with disabilities and the lack of studies supporting this issue, it seems that there is a need to conduct more studies focusing on the mental health of individuals with disabilities during the COVID-19 period. Therefore, this study examined the levels of pervasive mental health problems, including anxiety and depression, and social media exposure for individuals with disabilities during the COVID-19 pandemics in Saudi Arabia. This study also explored the impact of social media exposure on anxiety and depression among individuals with disabilities.

\section{The Research Questions}

- RQ1: What is the level of anxiety, depression, and social media exposure for individuals with disabilities?

- RQ2: How does social media exposure impact the anxiety and depression of individuals with disabilities during COVID-19?

\section{Methodology}

\subsection{Participants and Settings}

The current study conducted a quantitative method using a survey to collect data. An online questionnaire was distributed to the study participants after a month of prevailing COVID-19 in Taif, Saudi Arabia. This study was concurrently conducted with the release of previous restrictions implemented in Saudi Arabia. Although we expected 200 participants in this study, only 120 individuals with disabilities participated in the current study over five days. Also, simple random sampling was 
used to represent equal and independent selection for the sample. [See table 1]

Table 1. Characteristics of the participants

\begin{tabular}{|c|c|c|c|}
\hline \multicolumn{2}{|c|}{ Participants' characteristics } & \multicolumn{2}{|c|}{ Individuals with disabilities } \\
\hline & & Frequency & Percentage \% \\
\hline \multirow[t]{2}{*}{ Gender } & Male & 45 & 33.3 \\
\hline & Female & 75 & 54.7 \\
\hline \multirow[t]{5}{*}{ Age } & $15-25$ years & 32 & $25 \cdot 3$ \\
\hline & $26-35$ years & 44 & $33 \cdot 3$ \\
\hline & $36-50$ years & 22 & $17 \cdot 3$ \\
\hline & 51-6o years & 15 & $9 \cdot 3$ \\
\hline & Above 60 & 7 & 2.7 \\
\hline \multirow[t]{4}{*}{ Education } & Graduate & 25 & $9 \cdot 3$ \\
\hline & Bachelor & 46 & 40.0 \\
\hline & High School & 36 & 30.7 \\
\hline & less than high school & 13 & 8.0 \\
\hline \multirow[t]{3}{*}{ Health states } & Excellent & 25 & 10.7 \\
\hline & Very good & 25 & 10.7 \\
\hline & Good & 70 & 66.7 \\
\hline \multirow[t]{3}{*}{ Media exposure } & Rarely & 8 & 10.7 \\
\hline & Sometimes & 8 & 10.7 \\
\hline & Frequently & 50 & 66.7 \\
\hline
\end{tabular}

\subsection{Research Design and Procedures}

The cross-sectional approach, which is suitable to this study's nature, was used and conducted over five days $\left(1^{\text {st }}-5^{\text {th }}\right.$ June 2020) after active cases reached over 2000 cases during the Saudi government's curfew period. The study approval was obtained from the deanship of scientific research at Taif University and the administration of education in Taif, Saudi Arabia. The survey included an agreement question of participating in the study. The administration of education distributed the survey link in Taif as a text message. However, 120 questionnaires were returned.

\section{$5 \cdot 3$ Instrument}

Two reliable scales were engaged with minor adaptions in the current study. The first scale is Generalized Anxiety Disorder-7, " GAD-7," with a seven-item questionnaire identifying potential causes of generalized anxiety disorder and examining the severity of generalized anxiety disorder symptoms (Spitzer, Kroenke, Williams \& Löwe, 2006). Responses are ranged "Not at all," "Several days," "More than half a day," and "Almost every day" and numbered as 1, 2, 3, and 4, respectively. The scale's internal consistency was excellent (Cronbach $\alpha=.92$ ) (Spitzer et al., 2006). In the present research, GAD-7 was translated through a back translation, which is translated from English into Arabic, then from Arabic into English again. It was provided to experts in English and Arabic language to confirm the translation and language accurateness. Reliability was measured after collecting data; the consistency of the Saudi form of GAD-7 was good (Cronbach Alpha $=0.87$ ), consistent with previous studies, in which Cronbach Alpha ranged from 0.74 (Sidik, Arroll \& Goodyear-Smith, 2012) to 0.94 (García-Campayo et al., 2010).

Second, The CES-D-10 is a 10-item Likert scale survey assessing depression in the previous week. (CES-D-10) contains ten items distributed as follows three items for depressive consequence, five items for bodily symptoms, and two for positive consequence. Choices for each item are ranged from "not at all (1) to " almost every day" (4). Higher scores show a more considerable severity of symptoms. In the present study, CES-D-10 was translated through a back translation, which is translated from English into Arabic, then from Arabic into English again. It was provided to experts in English and Arabic 
languages to confirm the translation and language accurateness. Reliability was measured after collecting data; CES-D-1o revealed significant reliability and viability in the Saudi Arabian version, with good internal consistency in this study (Cronbach's $\alpha=.81$ ).

\subsection{Data analysis}

The data will be analyzed using descriptive and inferential statistics. The researchers used descriptive statistics, average and standard deviation through the analysis of SPSS 23. For the first question, we proceed to a descriptive analysis to measure the level of anxiety, depression, and media exposure. For the second question, we conducted a one-way ANOVA to investigate social media exposure on anxiety and depression of individuals with disabilities.

\section{Results}

Table 2. The average of anxiety, depression, and social media for individuals with disabilities

\begin{tabular}{lccc}
\hline & \multicolumn{3}{c}{ Individuals with disabilities } \\
\hline Media exposure & $\mathrm{N}$ & Mean & Std. Deviation \\
Total anxiety & 120 & 2.6364 & .69363 \\
Total depression & 120 & 3.3701 & .66911 \\
Valid N (listwise) & 120 & 3.5182 & .51914 \\
\hline
\end{tabular}

The current study aimed to reveal the two types of mental health problems: anxiety and depression, and their variation based on social media exposure of individuals with disabilities in Saudi Arabia, using a quantitative survey. The average scores of social media exposure, anxiety, and depression are pretty similar for individuals with disabilities. The average score of anxiety for individuals with disabilities is $(\mathrm{M}=3.37, \mathrm{SD}=.66)$. Also, the mean score of depression for individuals with disabilities is $(\mathrm{M}=3.51, \mathrm{SD}=$ $.51)$. However, the mean score of social media exposure for individuals with disabilities is $(\mathrm{M}=2.63, \mathrm{SD}=$ 2.63). [See table 2]

Table 3. Social media exposure and anxiety level

\begin{tabular}{lccccc}
\hline & Sum of Squares & $\mathrm{df}$ & Mean Square & $\mathrm{F}$ & Sig. \\
\hline Between Groups & 2.592 & 2 & 1.296 & 3.080 & .053 \\
Within Groups & 26.509 & 63 & .421 & & \\
Total & 29.101 & 65 & & & \\
\hline
\end{tabular}

We conducted a one-way ANOVA to investigate the effect of social media exposure on anxiety levels for individuals with disabilities, as measured by GAD-7. We also divided the outcomes of individuals with disabilities into three levels based on their social media exposure (level 1: Rarely; level 2: Sometimes; level 3: Frequently). we statistically found a significant variation at the level of $p<.05$ in average totals for the three age levels: $F(2,63)=3.08, p=.05$. We also noticed that the results were statistically significant; however, the average totals' actual difference between the levels was medium. The effect size considered employing eta squared scored .o8. When employing Post-hoc comparisons with Tukey test the average total for level $1(\mathrm{M}=3.83, S D=0.45)$ was statistically dissimilar to level 3 $(\mathrm{M}=3.34, S D=0.64)$. Level $2(\mathrm{M}=3.05, S D=0.78)$ did not change considerably from level 1 . [See table 3] 
Table 4. Social media exposure and depression level

\begin{tabular}{lccccc}
\multicolumn{7}{c}{ ANOVA } & & \\
\hline & Sum of Squares & df & Mean Square & F & Sig. \\
\hline Between Groups & 1.483 & 2 & .741 & 2.913 & .062 \\
Within Groups & 16.035 & 63 & .255 & & \\
Total & 17.518 & 65 & & \\
\hline
\end{tabular}

We conducted a one-way ANOVA to investigate the effect of social media exposure on depression levels for individuals with disabilities, as measured by CES-D-10. We also divided the outcomes of individuals with disabilities into three levels based on their social media exposure (level 1: Rarely; level 2: Sometimes; level 3: Frequently). We statistically found no significant variation at the level of $p<.05$ in average totals for the three age levels: $F(2,63)=2.91, p=.06$. We also noticed that the results were statistically significant; however, the average totals' actual difference between the levels was medium. The effect size considered employing eta squared scored .o8. When employing Post-hoc comparisons with Tukey test the average total for level $1(\mathrm{M}=3.85, S D=0.14)$ was statistically dissimilar to level 3 $(\mathrm{M}=3.34, S D=0.64)$. Level $2(\mathrm{M}=3.70, S D=0.61)$ did not change considerably from level 1. [See table 4]

\section{Discussion}

The current study's primary purpose is to reveal the level of anxiety, depression, and social media exposure for individuals with disabilities. The current results show a similar mean score of anxiety and depression, which is "more than a half-day" during the COVID-19 pandemic period for individuals with disabilities. Our results are in line with previous outcomes, which state that many general populations in Saudi Arabia had severe symptoms of anxiety and depression (Alkhamees et al., 2020). There was an elevation of stress and anxiety for parents playing multiple roles assisting their children during the COVID-19 epidemic period. (Dalton et al., 2020) Also, the current results revealed that individuals with disabilities are frequently exposed to negative information on social media during the COVID-19 pandemic period. This finding was similarly noted by (Goggin \& Ellis, 2020) who pointed out that individuals with disabilities had a frequent exposure to digital and social media during the COVID-19 pandemic which caused many threats to them.

The study also explored the impact of social media exposure on anxiety and depression for individuals with disabilities during the COVID-19 pandemics. The present research reveals that social media exposure has a significant impact on anxiety levels for individuals with disabilities but has no significant impact on depression levels for those individuals. The amount of social media exposure that the general population has during the COVID-19 epidemic period reveals misleading information increases anxiety and stress for many people (Dalton et al., 2020). The spreading of COVID-19 on the social web has threatened many individuals and made them anxious and stressed (Goggin \& Ellis, 2020).

The current study shows an increase in anxiety and depression as individuals with disabilities are anxious and depressed for more than a half-day, negatively impacting their daily lives. Thus, general recommendations about dealing with persons with disabilities can help them during the COVID-19 epidemic period. First, public health messages and languages must be clear and accessible for individuals with disabilities with sign language interpretations (Armitage \& Nellums, 2020). Moreover, mitigation measures should not lead to isolations for those individuals; instead, they should be protective. Also, healthcare providers should be exposed to a training program to increase their awareness of rights and dignity and safeguards against discrimination toward those individuals (Armitage \& Nellums, 2020). Essential lessons should be proposed to guide districts to overcome the educational challenges related to the COVID-19 pandemic (Tremmel et al., 2020). 


\section{Limitations and Suggestions}

This study was conducted in Taif city, Saudi Arabia, which has 65 cities distributed in 13 regions; therefore, it can be hard to generalize the study findings to the whole population. It is suggested to replicate this study in different regions of Saudi Arabia. The current study did not mainly examine the impact of demographic variables on anxiety, depression, and social media exposure for individuals with disabilities. This issue can be an avenue for future researches.

\section{Conclusion}

The current study mainly examined the pervasive mental health problems, including anxiety and depression, and social media exposure for individuals with disabilities. Individuals with disabilities feel anxious and depressed during the epidemic period, and they are frequently exposed to social media. Thus, the frequent exposure to misleading information on social media increased the anxiety level of individuals with disabilities but did not make them depressed. We suspected some variables such as age and health status could affect the increase of anxiety. This suggestion has to be noticed when replicating such a study in the future.

\section{Acknowledgement}

The authors would like to express their appreciation to the deanship of scientific research at Taif University, Saudi Arabia, for financing this study with the code number (1-441-6o).

\section{References}

Alkhamees, A. A., Alrashed, S. A., Alzunaydi, A. A., Almohimeed, A. S., \& Aljohani, M. S. (2020). The psychological impact of COVID-19 pandemic on the general population of Saudi Arabia. Comprehensive Psychiatry, 102, 152192 .

Armitage, R., \& Nellums, L .B. (2020). The COVID-19 response must be disability-inclusive. The Lancet Public Health, 5(5), e257.

Bartlett, J. D., Griffin, J., \& Thomson, D. (2020). Resources for supporting children's emotional well-being during the COVID-19 pandemic. Child Trends .12 ,

Boretti, A. (2020). COVID-19 fatality rate for Saudi Arabia, updated 3 June 2020. Journal of Global Antimicrobial Resistance, 22, 845-846.

Caton, S., \& Chapman, M. (2016). The use of social media and people with intellectual disability: A systematic review and thematic analysis. Journal of intellectual and developmental disability, 41(2), 125-139 .

Cocq, C., \& Ljuslinder, K. (2020). Self-representations on social media. Reproducing and challenging discourses on disability. Alter, 14(2), 71-84 .

Courtenay, K., \& Perera, B. (2020). COVID-19 and people with intellectual disability: impacts of a pandemic. Irish Journal of Psychological Medicine, 37(3), 231-236.

Dalton, L., Rapa, E., \& Stein, A. (2020). Protecting the psychological health of children through effective communication about COVID-19. The Lancet Child E Adolescent Health, 4(5), 346-347 .

Gale, F., \& Bolzan, N. (2016). Online ghettoes, perils or supernannies? Australian young people with chronic illness and disability challenge some moral panics about young people online. Disability E Society, 31(8), $1112-1126$.

García-Campayo, J., Zamorano, E., Ruiz, M. A., Pardo, A., Pérez-Páramo, M., López-Gómez, V., ... \& Rejas, J. (2010). Cultural adaptation into Spanish of the generalized anxiety disorder-7 (GAD-7) scale as a screening tool. Health and Quality of Life Outcomes, 8(1), 1-11.

Goggin, G., \& Ellis, K. (2020). Disability, communication, and life itself in the COVID-19 pandemic. Health Sociology Review, 29(2), 168-176.

Kowalski, R. M., Toth, A., \& Morgan, M. (2018). Bullying and cyberbullying in adulthood and the workplace. The Journal of social psychology, 158(1), 64-81.

Manganello, J. A. (2020). Media use for children with disabilities in the United States during COVID-19. Journal of Children and Media, 1-4. 
McNeil, K., Brna, P. M., \& Gordon, K. E. (2012). Epilepsy in the Twitter era: a need to re-tweet the way we think about seizures. Epilepsy E behavior, 23(2), 127-130 .

Miller, R .A. (2017). " My voice is definitely strongest in online communities": Students using social media for queer and disability identity-making. Journal of college student development, 58(4), 509-525 .

Patel, K. (2020). Mental health implications of COVID-19 on children with disabilities. Asian Journal of Psychiatry .

Sidik, S. M., Arroll, B., \& Goodyear-Smith, F. (2012). Validation of the GAD-7 (Malay version) among women attending a primary care clinic in Malaysia. Journal of Primary Health Care, 4(1), 5-11.

Spitzer, R. L., Kroenke, K., Williams, J. B., \& Löwe, B. (2006). A brief measure for assessing generalized anxiety disorder: the GAD-7. Archives of internal medicine, 166(10), 1092-1097.

National Center for Education Statistics, 2020. Children and Youth with Disabilities.

Retrieved from. https://nces.ed.gov/programs/coe/indicator_cgg.asp.

Sweet, K. S., LeBlanc, J. K., Stough, L. M., \& Sweany, N. W. (2020). Community building and knowledge sharing by individuals with disabilities using social media. Journal of computer assisted learning, 36(1), 1-11 .

Tandon, R. (2020). The COVID-19 pandemic, personal reflections on editorial responsibility. Asian Journal of Psychiatry, 50, 102100 .

Thelwall, M., \& Levitt, J. M. (2020). Re-tweeting Covid-19 disability issues: Risks, support and outrage. El profesional de la información (EPI), 29 .(2)

Tremmel, P., Myers, R., Brunow, D. A., \& Hott, B. L. (2020). Educating Students With Disabilities During the COVID-19 Pandemic: Lessons Learned From Commerce Independent School District. Rural Special Education Quarterly, 39(4), 201-210.

World Health Organization. (2020). Disability considerations during the COVID-19 outbreak (No. WHO/2019nCoV/Disability/2020.1). World Health Organization. 